\title{
NEUES AUS DER BIBLIOTHEKARISCHEN AUS- UND FORTBILDUNG IN DEN STUDIENJAHREN 2018/19 UND 2019/20
}

\author{
von Birgit Hörzer, Gabriele Pum, Alina Rezniczek, Monika Schneider-Jakob
}

\section{Inhalt}

1. Neues Curriculum 2019

1.1. Aktualisierte Inhalte

1.2. Blended Learning

1.3. Fachliche Spezialisierung

2. Aktuelle Lehrgänge

2.1. Grundlehrgang 2018/19

2.2. Grundlehrgang 2019-21

2.3. Masterlehrgang 2019-21

2.4. Abschlüsse 2018-19

3. Masterarbeiten 2019

4. Österreichweites Fortbildungsprogramm $4 L$ - Lifelong Learning for Librarians

\section{Neues Curriculum 2019}

\subsection{Aktualisierte Inhalte}

Ziel der Aktualisierung des Curriculums war es, der rasanten Entwicklung im Berufsfeld durch Anpassung der Ausbildungsinhalte und durch neue didaktische Konzepte Rechnung zu tragen, um den Lehrgang weiterhin attraktiv und flexibel zu gestalten. Die Absolventinnen und Absolventen sollten bestmöglich für den Berufseinstieg in einer Bibliothek oder Informationseinrichtung und den damit verbundenen Anforderungen vorbereitet sein.

Besonderes Augenmerk wurde inhaltlich auf die Bereiche Serviceorientierung, Technologieanwendungen und Informationsorganisation gelegt, ohne Praxisnähe und Konzentration auf die bibliothekarischen Kernkompetenzen zu vernachlässigen.

Die begleitende Berufspraxis sowie die Projektarbeit zählen weiterhin zu den besonderen Stärken des Lehrgangs. Sie sind als Vorbereitung auf den beruflichen Einstieg unverzichtbar - während des Praktikums können Studierende Lerninhalte vertiefen und ihre Einstiegschancen in den Beruf verbessern, darüber hinaus haben sie die Möglichkeit, wertvolle Netzwerke im Inund Ausland zu knüpfen. Im Rahmen der Projektarbeit lernen die Studieren- 
den mit Konzepten und Werkzeugen des Projektmanagements zu arbeiten und eine Fragestellung aus der Praxis im Team zu planen und umzusetzen.

\begin{tabular}{|l|c|c|}
\hline Grundlehrgang & SS & ECTS \\
\hline Bibliotheks- und Bestandsmanagement & 5 & 8 \\
\hline Informationsorganisation & 8 & 8 \\
\hline Informationstechnologie & 5 & 8 \\
\hline Informationsservices und Vermittlungskompetenz & 6 & 8 \\
\hline Projektmanagement & 4 & 8 \\
\hline Berufspraxis & - & 12 \\
\hline Wahlmodule & - & 8 \\
\hline Summe & 28 & 60 \\
\hline
\end{tabular}

Tab. 1: Abschluss „Akademische Bibliotheks- und Informationsexpertin“ bzw. „Akademischer Bibliotheks- und Informationsexperte“ 2019

\begin{tabular}{|l|c|c|}
\hline $\begin{array}{l}\text { Masterlehrgang } \\
\text { (60 ECTS werden im Rahmen des Gundlehrganges absolviert s.o.) }\end{array}$ & SS & ECTS \\
\hline Strategie und Management & 7 & 12 \\
\hline Wissensproduktion und Bibliothek & 5 & 8 \\
\hline Wahlmodule & - & 16 \\
\hline Master-Seminar & 2 & 2 \\
\hline Verteidigung der Masterthesis & - & 2 \\
\hline Masterthesis & - & 28 \\
\hline Summe & 28 & 60 \\
\hline
\end{tabular}

Tab. 2: Abschluss: Master of Science (Library and Information Studies) - MSc 2019

\subsection{Blended Learning}

Das Curriculum kombiniert klassische Unterrichtsmethoden mit innovativen elektronischen Lernformen (blended learning). Während der Präsenzmodule vermitteln die Lehrenden theoretische Grundlagen in Form von Gruppenarbeiten, Präsentationen, Diskussionen und Fallbeispielen. Soziale Interaktion und der Aufbau von beruflichen Netzwerken stehen neben der Vermittlung von Lehrinhalten im Vordergrund. 
In den Vor- und Nachbereitungsphasen bearbeiten die Studierenden unter Verwendung von Online Tools selbständig praxisnahe Themenstellungen. Diese neue Art des Kompetenzaufbaus stellt nicht nur für die Studierenden, sondern auch für die Vortragenden eine Neuorientierung dar. Die Betreuung reicht über die Präsenzphase hinaus und ermöglicht eine orts- und zeitunabhängige Interaktion zwischen Lehrenden und Lernenden. Dadurch ist erstmalig auch eine berufsbegleitende Durchführung des Lehrganges möglich.

\subsection{Fachliche Spezialisierung}

Ein weiterer Fokus wurde auf die fachliche Spezialisierung durch die Erhöhung des Angebotes an Wahlmodulen gelegt (s.u.), aus denen Studierende im Grund- sowie im Masterlehrgang je 8 ECTS zu absolvieren haben.

Zusätzlich werden einzelne Wahlmodule für die österreichweite fachliche Fortbildung im Rahmen des Weiterbildungsformats $4 \mathrm{~L}$ geöffnet. Damit ist es auch KollegInnen, deren Ausbildung schon längere Zeit zurückliegt möglich, kompakte Fortbildungen auf universitärem Niveau zu absolvieren.

\begin{tabular}{|l|c|c|}
\hline Wahlmodule Grundlehrgang & SS/KS & ECTS \\
\hline Spezialfragen zum Metadatenmanagement & 3 & 4 \\
\hline Angewandte Informationskompetenz & 3 & 4 \\
\hline Bibliotheksrelevante Rechtsfragen und Informationsethik & 2 & 4 \\
\hline Öffentliche Büchereien & 2 & 4 \\
\hline One Person Libraries (OPL) & 2 & 4 \\
\hline Nachlassverwaltung und Sondersammlungen & 3 & 4 \\
\hline Marketing und Öffentlichkeitsarbeit & 2 & 4 \\
\hline Aktuelle Schwerpunkte und Trends (Grundlehrgang) & 2 & 4 \\
\hline Archivkunde & 4 & 8 \\
\hline Data Librarian & 5 & 8 \\
\hline Historisches Buchgut & 5 & 8 \\
\hline
\end{tabular}

Tab. 3: Übersicht über die Möglichkeiten der fachlichen Spezialisierung im Rahmen des Grundlehrganges 


\begin{tabular}{|l|c|c|}
\hline Wahlmodule Masterlehrgang & SS/ KS & ECTS \\
\hline Bibliotheksarchitektur & 2 & 4 \\
\hline Bibliometrie und Szientometrie & 3 & 4 \\
\hline Rechtspraxis in Bibliotheken & 4 & 4 \\
\hline Innovations- und Change-Management in Bibliotheken & 2 & 4 \\
\hline Bibliotheksspezifische Zielgruppenforschung & 2 & 4 \\
\hline Strategisches Management in Öffentlichen Büchereien & 2 & 4 \\
\hline Aktuelle Schwerpunkte und Trends (Masterlehrgang) & 2 & 4 \\
\hline
\end{tabular}

Tab. 4: Übersicht über die Möglichkeiten der fachlichen Spezialisierung im Rahmen des Masterlehrganges

\section{Aktuelle Lehrgänge}

\subsection{Grundlehrgang $2018 / 19$}

Im September 2019 schlossen 22 Studierende an der Österreichischen Nationalbibliothek und 25 an der Universitätsbibliothek Wien den Grundlehrgang des Universitätslehrgangs Library and Information Studies erfolgreich $a b$.

Abschlussprojekte an der Österreichischen Nationalbibliothek:

- Grundlagen für die Erstellung eines Bibliotheksentwicklungsplanes für das Burgenland

- Neuorganisation der Bibliothek des Vereins Selbstlaut

- VÖB Webpräsenz reloaded

- Virtuelles Portal für die Privilegiensammlung im Österreichischen Patentamt

- Feinerschließung des Teilnachlasses von Konrad Mautner (18801924) im Archiv des Österreichischen Volksliedwerkes

- Kontrolliertes Vokabular ZAMG

- Bibliothekarische Erschließung des protestantischen Sonderbestandes der Stiftsbibliothek Göttweig

- „Biblioteca Capucinorum Braunau“ - Provenienzerschließung der Kapuzinerbibliothek Braunau

- Sar goes GND 
Abschlussprojekte an der Universitätsbibliothek Wien:

- Acquisitionsbuch der UB Wien

- Bearbeitung des philosophischen Nachlasses von Univ.-Prof. Dr. Falk Wagner

- Die Klaar-Pläne - Bestandsnachweis und Archivierung

- Digitale Datenarchive und Barrierefreiheit

- Paul F. Lazarsfeld Archiv

- Varianten für eine Neuaufstellung des Zeitschriften- und ReihenBestandes der FB Klassische Archäologie und der Bibliothek Wien des ÖAl (ÖAW)

- Zeitschriftendepotbewirtschaftung

\subsection{Grundlehrgang 2019-21}

Im WS 2019/20 startete der erste Grundlehrgang des Universitätslehrgangs Library and Information Studies nach Curriculum 2019 an der Universitätsbibliothek Wien mit 27 Studierenden, an der Österreichischen Nationalbibliothek mit 22 Studierenden und an der Universitäts- und Landesbibliothek Tirol mit 20 Studierenden.

\subsection{Masterlehrgang 2019-21}

Im Oktober 2019 startete - ebenfalls nach Curriculum 2019 - der erste Masterlehrgang mit 13 Studierenden an der Universität Wien in Kooperation mit der Österreichischen Nationalbibliothek.

\subsection{Abschlüsse 2018-19}

\begin{tabular}{|l|l|c|c|}
\hline Ausbildung & Qualifikation & 2018 & 2019 \\
\hline $\begin{array}{l}\text { Grundlehrgang Library } \\
\text { and Information Studies }\end{array}$ & Matura & 45 & 47 \\
\hline $\begin{array}{l}\text { Masterlehrgang Library } \\
\text { and Information Studies }\end{array}$ & Hochschulabschluss & 3 & 3 \\
\hline $\begin{array}{l}\text { Lehrgang Bibliothek, Information } \\
\text { und Dokumentation }\end{array}$ & $\begin{array}{l}\text { Lehre, } \\
\text { Berufsschulabschluss }\end{array}$ & 13 & - \\
\hline $\begin{array}{l}\text { Lehrabschluss Archiv-, Bibliotheks- } \\
\text { und Informationsassistentln }\end{array}$ & - & 28 & 13 \\
\hline
\end{tabular}




\section{Masterarbeiten 2019}

Eine Liste aller seit 2004 verfassten Masterarbeiten findet sich unter: https://www.bibliotheksausbildung.at.

\section{Mag. ${ }^{a}$ Romana Holtemayer, MSc: Zum zeichenhaften Gebrauch des verbote- nen Buches als Transportmittel unerwünschten Gedankenguts im Film - eine dramaturgische Analyse anhand ausgewählter Spielfilme}

Diese Master-Thesis befasst sich mit dem zeichenhaften Gebrauch und der dramaturgischen Funktion des Buchobjektes als Träger von Information im Spielfilm. Das Buch dient bereits seit Jahrhunderten als Symbol für Wissen und Bildung, es konnte seinem Besitzer Prestige und Macht verleihen. Die über dieses traditionsreiche Medium übermittelte Information wurde aber mitunter auch als unliebsam und sogar "gefährlich" eingestuft. Zensorische Maßnahmen, die bis zur Vernichtung des Buchkörpers reichen konnten, prägten vergangene Epochen, die Bücherverbrennung als symbolischer Akt der Tilgung unerwünschter Ideen oder von der eigenen Weltsicht abweichender Gedanken findet aber auch im 21. Jahrhundert noch Anhänger.

Seine singuläre Stellung als Leitmedium hat das Buch im 20. Jahrhundert eingebüßt, doch der Medienwechsel ermöglicht eine Betrachtung des alten Mediums im Spiegel neuerer Medien. Der Spielfilm steht schon sehr früh, seit seiner Entwicklung zum Erzählkino, in einer Wechselbeziehung zu Literatur und literarischem Theater. Doch erst durch den Aufstieg des Fernsehens und besonders durch den Einfluss digitaler Medien ist ab Mitte des 20. Jahrhunderts eine intensiver werdende Beschäftigung mit der Buchkultur beziehungsweise deren drohendem Ende zu verzeichnen. Dieses Thema wird selbstreflexiv in der Literatur verarbeitet, findet seinen Weg aber auch vermehrt auf die Kinoleinwand.

Im Film wird das Buch als Objekt im Kodex-Format inszeniert, selten sieht oder hört man Textpassagen, selten wird man mit dem konkreten Inhalt eines Buches konfrontiert. Seine „Rolle“ im Film ist aber immer eindeutig interpretierbar - hier sind wichtige Informationen gespeichert, Fakten, keine „fake news“. Als dramaturgischer Höhepunkt der Spielfilmhandlung fungiert häufig ein spektakulärer Bibliotheksbrand, der aber auch klärende und aufrüttelnde Wirkung haben kann. Anhand der ausgewählten Filmbeispiele aus unterschiedlichen Jahrzehnten wird die Bandbreite des Themas sichtbar, die zwischen nostalgischer Auratisierung der Buchkultur und der Realisierung des bereits vollzogenen Medienwandels schwankt. Gemein- 
sam ist allen Inszenierungen jedenfalls, dass Büchern und dem durch sie transportierten Wissen große Macht über den Menschen zugeschrieben wird.

\section{Mag. ' Julia Langmeier, MSc: Der Umgang mit Buchnachlässen an kirchlich- wissenschaftlichen Bibliotheken am Beispiel der Diözesanbibliothek Würzburg}

Die Arbeit widmet sich der Bedeutung von Buchnachlässen und ganzen Nachlassbibliotheken für kirchlich-wissenschaftliche Bibliotheken in Deutschland und Österreich. Nach einer kurzen bibliothekswissenschaftlichen Übersicht über den Stand der Forschung zu Nachlassbibliotheken wird ausgehend von der speziellen Situation und den Workflows der Diözesanbibliothek Würzburg analysiert, welche Personenkreise ihre Bibliotheken vererben oder schon zu Lebzeiten verschenken, welche Bedeutung diesen Buchnachlässen innerhalb des Gesamtbestandes zukommt und wie diese besonderen Bestände in den jeweiligen Institutionen verwaltet, in den Gesamtbestand integriert und auch erforscht werden. Ziel der Masterarbeit ist es, die Vielfalt an unterschiedlichsten Nachlassbibliotheken aufzuzeigen, ihre Bedeutung für das kirchlich-wissenschaftliche Bibliothekswesen herauszuarbeiten und nicht zuletzt aus diesem Vergleich mit anderen Institutionen ein spezielles Best-Practice-Modell für den Umgang mit Buchnachlässen an der Diözesanbibliothek Würzburg zu entwickeln. Die Arbeit gliedert sich in drei große Abschnitte: Der erste, historisch-theoretische Teil gibt einen Überblick über die Geschichte der Forschungen zu Nachlassbibliotheken, Begriffsdefinitionen, Regelwerken und (auch kirchlichen) Leitlinien sowie Präsentationsmethoden. Danach wird die wechselvolle Geschichte der Diözesanbibliothek Würzburg und ihrer Buchnachlässe vorgestellt, woran sich ein Überblick über die dokumentierten nachgelassenen Buchbestände und die bisherige Bearbeitungspraxis anschließt. Darauf folgt der zweite, praktische Teil der Thesis, in dem eine die Masterthesis begleitende Onlineumfrage vorgestellt und ausgewertet wird. Ziel dieser Umfrage ist ein Einblick in Provenienzen, Erwerb, Erschließungs- und Verwaltungspraxis bei Buchnachlässen an kirchlich-wissenschaftlichen Bibliotheken in Österreich und Deutschland gewesen. Im abschließenden normativen Abschnitt der Arbeit wird anhand von Beispielen aus anderen Institutionen sowie den Erkenntnissen aus der Online-Umfrage eine Reihe von Empfehlungen sowie ein verbesserter Workflow für den Umgang mit Buchnachlässen an der Diözesanbibliothek Würzburg entwickelt. Mit der Untersuchung der Bedeutung von Buchnachlässen für kirchlich-wissenschaftliche Bibliotheken vermag die Arbeit zum einen, eine Lücke in der Forschung zu Nachlassbibliotheken zu 
schließen, zum anderen liefert sie einen Beitrag zur Geschichte der Diözese und der Institution Archiv und Bibliothek des Bistums Würzburg.

\section{Mag. ${ }^{a}$ Andrea Torggler, MSc: Publikationsunterstützende Services an Biblio- theken - eine Chance für österreichische Fachhochschulen}

Österreichische Fachhochschulen versuchen sich in den letzten Jahren verstärkt im Bereich Forschung zu positionieren. Bedingt durch ihre anwendungsorientierte Ausrichtung und ihre Finanzierung haben sie dabei komplett andere Voraussetzung als die Österreichischen Universitäten. Möchte eine Fachhochschule erfolgreich Forschungsagenden betreiben, müssen gezielt Prioritäten und Maßnahmen innerhalb der Institution gesetzt werden. Bedingt durch Veränderungen des digitalen Informationszeitalters, bemühen sich auch Hochschulbibliotheken zunehmend, neben den klassischen etablierten Bibliotheksaufgaben Forscherlnnen und ihre Bedürfnisse in den Fokus ihrer Dienstleistungen zu stellen. Fachhochschulbibliotheken in Österreich haben hier jedoch noch kaum Angebote. Mit einer Umfrage unter den österreichischen Fachhochschulbibliotheken und einem Vergleich der Kennzahl „Publikation“ in den jeweiligen Jahresberichten wird ein Überblick über den Status Quo der forschungsunterstützenden Services der Bibliotheken an Fachhochschulen erstellt. Die dargelegten Defizite können als Chance gesehen werden: Neben der Erhebung der Publikationskennzahlen eignen sich noch einige andere publikationsunterstützende Tätigkeiten besonders gut, um die Forschungsagenden der Fachhochschule nachhaltig zu fördern. Als ExpertInnen im Bereich von Daten- und Informationskompetenz wären Bibliotheken die effizientesten Abteilungen der Fachhochschule, um Wissen an zentraler Stelle aufzubauen und in der Organisation beständig weiter zu geben. Im Idealfall wird diese zur institutionellen Anlaufstelle für Forschungsfragen und die Fachhochschule könnte Forschungsagenden schneller aufbauen, dauerhaft sichern und auch selbstbewusst nach außen kommunizieren. Die Stärkung der Abteilung Fachhochschulbibliothek könnte also eine Strategie sein, die für alle Beteiligten vorteilhaft ist.

\section{4. Österreichweites Fortbildungsprogramm 4L - Lifelong Learning for Librarians}

In einem so dynamischen Berufsumfeld wie dem Bibliothekswesen braucht es eine ständige Auseinandersetzung mit aktuellen Inhalten. Deshalb haben sich die Universitätsbibliotheken Wien, Graz und Innsbruck mit der 
Österreichischen Bibliothekenverbund und Service Gesellschaft m. b. H. (OBVSG) und der Vereinigung Österreichischer Bibliothekarinnen und Bibliothekare (VÖB) darüber verständigt, ein gemeinsames Konzept für die bibliothekarische Weiterbildung zu erstellen und umzusetzen.

Das praxisnahe Weiterbildungsprogramm 4L - Lifelong Learning for Librarians startete im Februar 2019 und sieht einerseits eintägige Kurse zu aktuellen bibliothekarischen Fragestellungen vor, andererseits werden nach Maßgabe von freien Plätzen auch die Wahlfächer des Universitätslehrgangs Library and Information Studies sowie die Module der Zertifikatskurse für die Fortbildung geöffnet.

Das Programm lebt und kann auch kurzfristig angepasst werden, Kurse finden sowohl in Wien als auch in Graz und Innsbruck statt.

Insgesamt fanden seit Februar 201920 Kurse an der Universitätsbibliothek Wien, 13 an der Universitätsbibliothek Graz und 5 an der Universitäts- und Landesbibliothek Tirol statt.

Weitere Informationen finden sich auf der Homepage der Arbeitsgemeinschaft der Ausbildungsverantwortlichen: https://www.bibliotheksausbildung.at.

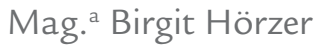
Universität Graz, Universitätsbibliothek E-Mail: birgit.hoerzer@uni-graz.at

Dr. ${ }^{\text {in }}$ Gabriele Pum Österreichische Nationalbibliothek E-Mail: gabriele.pum@onb.ac.at

Mag. ${ }^{a}$ Alina Rezniczek Universität Wien, Bibliotheks- und Archivwesen E-Mail: alina.rezniczek@univie.ac.at

Mag. ${ }^{a}$ Monika Schneider-Jakob, MAS Universität Innsbruck, Universitäts- und Landesbibliothek Tirol E-Mail: monika.schneider-jakob@uibk.ac.at

DOI: https://doi.org/10.31263/voebm.v73i1.3414

(c) Birgit Hörzer, Gabriele Pum, Alina Rezniczek, Monika Schneider-Jakob Dieses Werk ist - exkl. einzelner Logos und Abbildungen - lizenziert unter einer Creative-Commons-Lizenz Namensnennung 4.0 International 